Noname manuscript No.

(will be inserted by the editor)

\title{
Comprehensive Analysis of Market Conditions in the Foreign Exchange Market
}

\section{Fluctuation Scaling and Variance-Covariance Matrix}

\author{
Aki-Hiro Sato, Takaki Hayashi, and Janusz A. \\ Hołyst
}

Received: 24 September 2010 / Accepted: 3 March 2012

\begin{abstract}
We investigate quotation and transaction activities in the foreign exchange market for every week during the period of June 2007 to December 2010. A scaling relationship between the mean values of number of quotations (or number of transactions) for various currency pairs and the corresponding standard deviations holds for a majority of the weeks. However, the scaling breaks in some time intervals, which is related to the emergence of market shocks. There is a monotonous relationship between values of scaling indices and global averages of currency pair cross-correlations when both quantities are observed for various window lengths $\Delta t$.
\end{abstract}

Keywords Foreign Exchange Market · Fluctuation Scaling · Scaling Breaking • Global Average of Cross-Correlations

PACS 89.65.Gh $\cdot 02.50 . \mathrm{Cw}$

\section{Introduction}

Recent development of information and communication technology empowers us both to collect and analyse a large amount of data on socio-economic systems. Several researchers refer to the present epoch driven by this technological changes as the information explosion era 1 .

A.-H. Sato

Graduate School of Informatics, Kyoto University, Yoshida Honmachi, Sakyo-ku, Kyoto, 6068501, JAPAN

Tel.: +81-75-753-5515

Fax: +81-75-753-4919

E-mail: aki@i.kyoto-u.ac.jp

T. Hayashi

Graduate School of Business Administration, Keio University

4-1-1 Hiyoshi, Yokohama, Kanagawa 223-8526, JAPAN

E-mail: takaki@kbs.keio.ac.jp

J.A. Hołyst

Faculty of Physics and Center of Excellence for Complex Systems Research,

Warsaw University of Technology, Koszykowa 75, PL-00-662, Warsaw, POLAND

E-mail: jholyst@if.pw.edu.pl 
This progress enables us to monitor many details of financial assets and to record the information as a high frequency multivariate time series.

Recently, investigations of foreign exchange rates have been conducted by numerous researchers using various approaches based on statistical physics and time series analysis $2,3,4,5,6,7,8,9,10,11$. Drożdż et al. showed that exchange rate return fluctuations for main currency pairs are well described by non-extensive statistics and possess multifractal characteristics 2. Rebitzky studied the influence of macroeconomic news on exchange rates [3]. He concluded the following three points: (1) fundamental news do matter, (2) news influences exchange rates by incorporating common information into prices directly and indirectly based upon order flow and (3) the impact of fundamental news on exchange rates is fairly stable over time. Kaltwasser 4 estimated the herding tendency in the foreign exchange market for three currency pairs using the extended Alfrano-Lux model [5] and computed several unconditional moments of corresponding daily log-returns. Gworek et al. analysed the exchange rate returns of 38 currencies (including gold) 6]. They examined the cross-correlations between the returns of various currency pairs as well as between their signs, and in this way, they constructed a corresponding Minimal Spanning Tree for several base currencies. Liu et al. applied cross-sample entropy (Cross-SampEn) to compare two different time series in order to asses their degree of asynchrony to the daily log-return time series of foreign exchange rates in the currency markets [7. They compared the correlations between time series with Cross-SampEn and showed that Cross-SampEn is superior in describing the correlations.

Moreover, theoretical models 8, 9, and empirical analysis 10,11 on the tick-bytick data of the foreign exchange market have been considered. Ohnishi et al. 10] proposed a weighted-moving-average analysis for the tick-by-tick data of yen-dollar exchange rates. They concluded that the weights decay exponentially with a time scale less than $2 \mathrm{~min}$, implying that dealers are watching only the very recent market state. Hashimoto and Ito examined market impact of Japanese macroeconomic statistic news within minutes of their announcements on the yen-dollar exchange rates [1]. They found clear increases in the number of deals and price volatility immediately after such announcements.

In principle, information arrivals can be never observed comprehensively; however, one can register quotations(transactions) from market participants. Perceived information determines investor attitude, but the impact of news on trading decisions is an open question [12].

Nevertheless, we believe that it is important to confirm whether correlations exist between the number of quotations and transactions. In this study, we focus on the number of quotations or transactions for several currency pairs quoted in the foreign exchange market and we treat them as a multivariate time series that characterise system dynamics. Bonanno et al. investigated the spectral density of both the logarithm of prices and the daily number of trades of a set of stocks traded in the New York Stock Exchange 13. They confirmed the $1 / f^{2}$ behaviour of the spectral density of stocks log-returns and detected a $1 / f$-like behaviour of the spectral density of daily trade numbers. This shows that the number of transactions is generated by a long memory process, similar to the volatility dynamics.

More conceptually, the mixture distribution hypothesis is one of the most famous explanatory models for price fluctuations in the financial market 14, 15, 16, 17, 18, 19. Specifically, there is a positive simultaneous correlation between transaction volume, or the number of transactions, and returns [14,18,19. Furthermore, Mandelbrot- 
Taylor [14 and Clark [15] proposed the concept of time changes or subordinated processes in order to explain the fat-tailedness of returns. It was reported that the number of trades is profoundly related to volatility. According to the normal variance mixture models in finance [20], despite of the heteroskedacity of volatility with long memory, unconditional distribution of log-returns can be fitted to the mixture of normal distributions with an unconditional distribution of volatility. The same theory has been recently proposed in statistical physics by Beck to investigate superstatistics [21. Therefore, it is important to capture the cross-sectional structure of quotations and transactions to understand their volatility.

In studies on financial time series, several researchers have taken the stance that traded volume, or the number of transactions (quotations), is a proxy variable for unobservable information arrivals on market participants [15, 16, 18].

Researchers of econophysics focus on several types of scaling relationships observed in the financial markets in order to understand the behaviour of market participants 22 , $23,24,25$. Specifically, a scaling relationship between mean values of money flow and their standard deviation ("Taylor's power law" or "fluctuation scaling") is a useful scaling relationship when conducting multi-dimensional analysis on financial markets.

A scaling relationship between a mean of constituents' flows at the $i$-th observation point $\mu_{i}$ and their standard deviation $\sigma_{i}$ is referred to as fluctuation scaling:

$$
\mu_{i}=A \sigma_{i}^{\alpha},
$$

where $A$ represents a positive constant, and $\alpha$ is a scaling exponent taking a value ranging from $1 / 2$ to 1 . The pioneering work on fluctuation scaling is known as a Taylor's study in ecology [26]. After his study, the scaling relationship between means and standard deviations has been found in a wide spectrum of fields. Recently, Menezes and Barábasi have reported the scaling relationship on traffic flows, river flows and browsing activities of Internet 27. Eisler and Kertész investigated fluctuation scaling of the traded value and dependence of the scaling exponent on a time scale 25. More recently they computed the scaling relationship of the traded volume for 2647 stocks listed in the New York Stock Exchange (NYSE) 28] and 4039 listed in the NASDAQ in the TAQ database, which records all transactions of the NYSE and NASDAQ during the period from 2000 to 2002 . They found a clear scaling relationship at these stock markets. They clarified that the Hurst exponents and the scaling exponents depend on the window length. Eisler et al. investigated fluctuation scaling on the volumes traded in the stock exchange market.

Furthermore, some researchers have examined cross-correlations among various quantities of the financial markets 29, 30,31,32. Podobnik et al. investigated volume growth rate and volume changes for 14,981 daily records of the Standard and Poor's (S\&P) 500 index over a 59-year period (1950-2009) [30]. Using detrended crosscorrelation analysis, they found that there are power-law cross-correlations between them. Bonanno et al. studied correlation-based network analysis of financial equities 33 .

However, it is not obvious whether a stable scaling relationship exists in the foreign exchange market. This is the first focus of our paper. Following the Taylor methodology, we examine the scaling relationship between the mean of the numbers of quotations and transactions and their variance.

We suppose that there should exist a relationship between fluctuation scaling for the market activity and cross-correlations at the foreign exchange market. This is the second focus of this article. 
This article is organised as follows. In Sec. 2, we analyse the scaling exponents and cross-correlation matrices for a selected period. In Sec. 3, we conduct a long-term analysis of both the scaling exponents and the global average of the cross-correlations of both quotation and transaction numbers. Sec. 4 is devoted to concluding remarks and used to address future works.

\section{The scaling exponent and global average of correlations}

In this section, we focus on the number of quotations arriving at the matching engine of the EBS Platform and the number of transactions occurring there per unit time. We use comprehensive data (ICAP EBS Data Mine Level 1.0) collected by the EBS Platform, one of the most popular electronic brokerage systems, which is exploited by over 2,000 traders at about 800 dealing rooms across the globe [34. The data include records for orders (BID/OFFER) and transactions of currencies and precious metals during a period from June 2007 to December 2010 (43 months) 11. During this period, the global financial system suffered from the following significant macroeconomic shocks and crises: (I) Paribas shock (Aug. 2007), (II) Bear Stearns shock (Feb. 2008), (III) sub-prime crisis driven by Lehman shock (Sep. 2008 to Mar. 2009) and (IV) Euro crisis (Apr. to May 2010).

Let $P_{j, \Delta t}(k)$ and $D_{j, \Delta t}(k)$ be the number of quotations and transactions for exchange between the pairwise currencies $j$ during an observation period between $k \Delta t$ and $(k+1) \Delta t(k=0,1, \ldots, Q-1)$, respectively. Let us further assume that $P_{j, \Delta t}(k)$ and $D_{j, \Delta t}$ are locally stationary variables in the time interval $[0,(Q-1) \Delta t]$. A temporal mean and a variance-covariance matrix are expressed by the notation $\langle\cdots\rangle$ and $\operatorname{Cov}(\cdot, \cdot)(\tau)$ :

$$
\begin{aligned}
\left\langle X_{j, \Delta t}\right\rangle & =\frac{1}{Q} \sum_{k=0}^{Q-1} X_{j, \Delta t}(k), \\
\operatorname{Cov}\left(X_{i, \Delta t}, Y_{j, \Delta t}\right)(\tau) & =\left\{\begin{array}{l}
\frac{1}{Q+\tau} \sum_{k=0}^{Q+\tau-1}\left(X_{i, \Delta t}(k-\tau)-\left\langle X_{i, \Delta t}\right\rangle\right)\left(Y_{j, \Delta t}(k)-\left\langle Y_{j, \Delta t}\right\rangle\right)(\tau<0) \\
\frac{1}{Q-\tau} \sum_{k=0}^{Q-\tau-1}\left(X_{i, \Delta t}(k)-\left\langle X_{i, \Delta t}\right\rangle\right)\left(Y_{j, \Delta t}(k+\tau)-\left\langle Y_{j, \Delta t}\right\rangle\right)(\tau \geq 0)
\end{array},\right.
\end{aligned},
$$

where $X$ and $Y$ are selected from the set consisting of $P$ and $D$.

1 We found 43 kinds of currencies and 68 currency pairs during the whole observation period: AUD/NZD, AUD/USD, CHF/JPY, EUR/CHF, EUR/CZK, EUR/DKK, EUR/GBP, EUR/HUF, EUR/ISK, EUR/JPY, EUR/NOK, EUR/PLN, EUR/SEK, EUR/SKK, EUR/USD, EUR/ZAR, GBP/USD, NZD/USD, USD/CAD, USD/CHF, USD/HKD, USD/JPY, USD/MXC, USD/MXN, USD/MXT, USD/PLN, USD/RUB, USD/SGD, USD/ZAR, XAG/USD, XAU/USD, XPD/USD, XPT/USD, GBP/JPY, GBP/CHF, USD/TRY, XAU/JPY, AUD/JPY, USD/THB, CAD/JPY, NZD/JPY, EUR/RUB, ZAR/JPY, EUR/AUD, EUR/CAD, EUR/RON, GBP/AUD, USD/SEK, BKT/RUB, USD/DKK, USD/NOK, USD/ILS, SAU/USD, DLR/KES, DLR/KET, USD/AED, USD/BHD, USD/KWD, USD/SAR, EUQ/CHF, EUQ/JPY, USQ/CHF, EUQ/USD, USQ/JPY, USD/CNH, CNH/HKD, CNH/JPY and EUR/CNH. The total number of both quotations and transaction recorded during this observation duration is $318,871,428$. 
Elements of the correlation matrix of both quotation or transaction activities for various currency pairs $i$ and $j$ are, respectively, defined in the standard way:

$$
C_{i j, \Delta t}^{(X X)}(\tau)=\frac{\operatorname{Cov}\left(X_{i, \Delta t}, X_{j, \Delta t}\right)(\tau)}{\sqrt{\operatorname{Cov}\left(X_{i, \Delta t}, X_{i, \Delta t}\right)(\tau) \operatorname{Cov}\left(X_{j, \Delta t}, X_{j, \Delta t}\right)(\tau)}} .
$$

The mean value of its nondiagonal components describes the global average of simultaneous cross-correlation coefficients:

$$
\left\langle C_{\Delta t}^{(X)}\right\rangle=\frac{2}{N(N-1)} \sum_{i=1}^{N-1} \sum_{j=i+1}^{N} C_{i j, \Delta t}^{(X X)}(0),
$$

where $X=P$ for quotations and $X=D$ for transactions.

We shall look for a scaling relationship in the form:

$$
\left\{\begin{array}{l}
\operatorname{Cov}\left(P_{i, \Delta t}, P_{i, \Delta t}\right)(0)=A_{P}\left\langle P_{i, \Delta t}\right\rangle^{2 \alpha_{P}} \\
\operatorname{Cov}\left(D_{i, \Delta t}, D_{i, \Delta t}\right)(0)=A_{D}\left\langle D_{i, \Delta t}\right\rangle^{2 \alpha_{D}}
\end{array}\right.
$$

where $A_{P}$ and $A_{D}$ are positive constants and $\alpha_{P}$ and $\alpha_{D}$ are scaling exponents ranging from $1 / 2$ to 1 . This scaling law means that currency pairs with a low (high) mean activity show small (large) activity fluctuations. Values of scaling exponents $\alpha_{X}$ can be estimated by using the least squared method applied to the equation:

$$
\log \operatorname{Cov}_{X X}\left(X_{i, \Delta t}, X_{i, \Delta t}\right)(0)=\log A_{X}+2 \alpha_{X} \log \left\langle X_{i, \Delta t}\right\rangle .
$$

Figure 1 shows double logarithmic plots for standard deviations of quotations or transactions numbers against their mean values for investigated currency pairs during a selected period. The error bars represent 2-bootstrap standard deviations calculated from 1,000 bootstrap samples [35. In Ref [36], two of the authors (A.-H. Sato and J.A. Hołyst) investigated the scaling relationship with a different database of the foreign exchange market and found fluctuation scaling for quotation activities. Similarly, one can observe that the scaling Eq. (1) is well fulfilled with the ICAP database.

Figure 2 shows the dependence of $\alpha_{P}$ and $\left\langle C_{\Delta t}^{(P)}\right\rangle$ on the values of $\Delta t$ and a scatter plots of $\alpha_{P}$ and $\left\langle C_{\Delta t}^{(P)}\right\rangle$ at different values of $\Delta t$. Both $\alpha_{P}$ and $\left\langle C_{\Delta t}^{(P)}\right\rangle$ increase as $\Delta t$ increases.

Moreover, we extend the global average of cross-correlation coefficients between the number of quotations and transactions. By using Eq. (3), we define the cross-correlation function matrix as

$$
P D_{i j, \Delta t}(\tau)=\frac{\operatorname{Cov}\left(P_{i, \Delta t}, D_{j, \Delta t}\right)(\tau)}{\sqrt{\operatorname{Cov}\left(P_{i, \Delta t}, D_{i, \Delta t}\right)(\tau) \operatorname{Cov}\left(P_{j, \Delta t}, D_{j, \Delta t}\right)(\tau)}},
$$

and the global average of cross-correlations as

$$
\left\langle P D_{\Delta t}\right\rangle(\tau)=\frac{1}{N^{2}} \sum_{i=1}^{N} \sum_{j=1}^{N} P D_{i j, \Delta t}(\tau) .
$$

Obviously $P D_{i j, \Delta t}(\tau)$ quantifies the anticipatory behaviour of the quotation activities against transaction activities.

Figure 3 shows the global average of the cross-correlations between the quotation activities and transaction activities. One can see that the global average of the crosscorrelations possesses a maximum for the time difference $\tau=0$. Further, the positive tail of the cross-correlation function decays slower in time than does the negative one. 


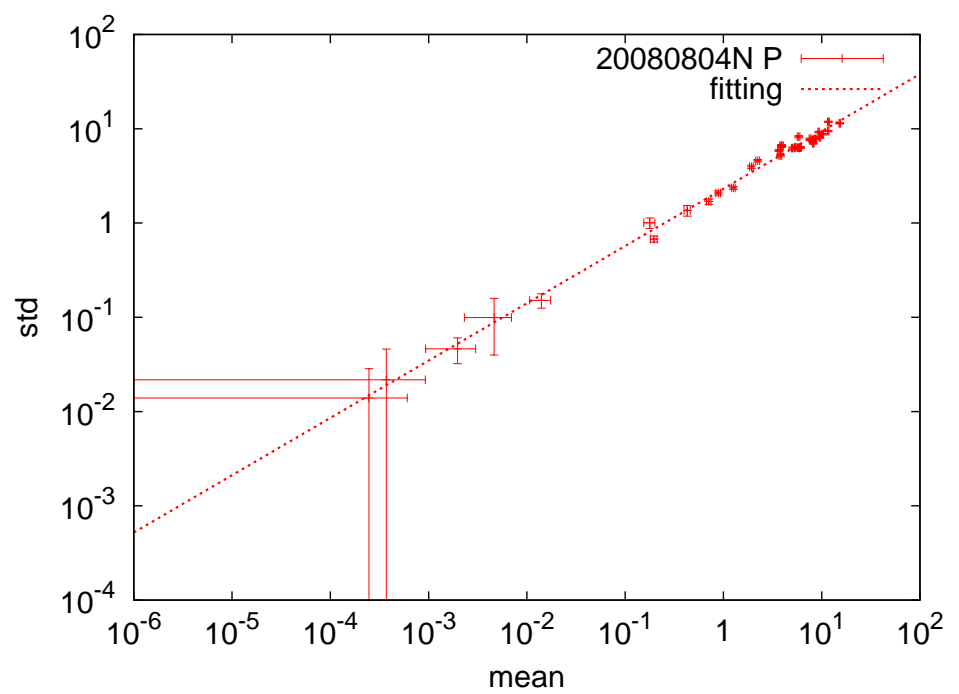

$(\mathrm{P})$

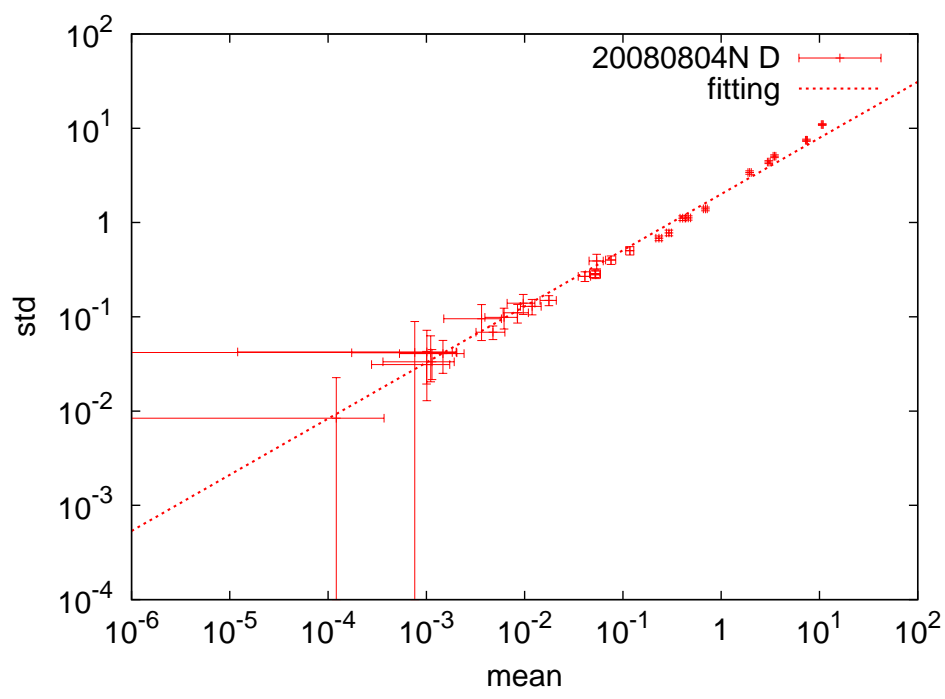

(D)

Fig. 1 The double logarithmic plots mean of the number of quotations $(\mathrm{P})$ and transactions (D) and their standard deviation. We use high resolution data from 8:38, 3 August 2008, to 21:56, 8 August 2008 (UTC). The error bars show 2-bootstrap standard deviations.

\section{Long-term analysis}

Now we show results for longer time periods. Using ICAP EBS Data Mine Level 1.0, we performed the investigations of the scaling exponent and the global average of crosscorrelations for both quotation and transaction activities. Throughout this analysis, we fix $T=Q \Delta t=10,080[\mathrm{~min}]$ and $\Delta t=1[\mathrm{~min}]$. The empirical results are obtained from Eqs. (2) and (3). 


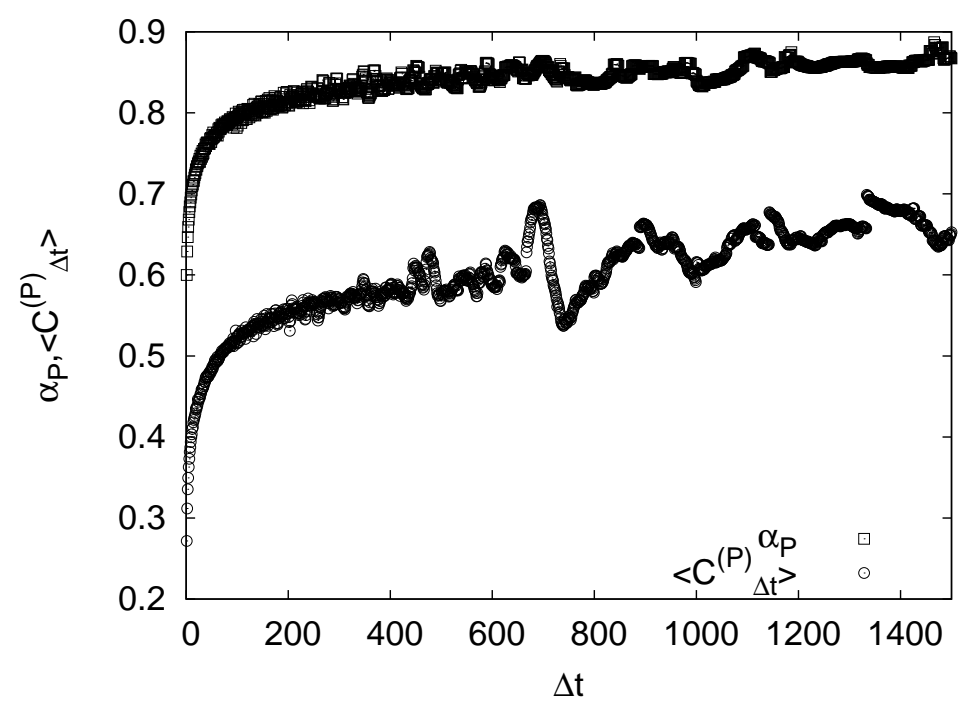

(a)

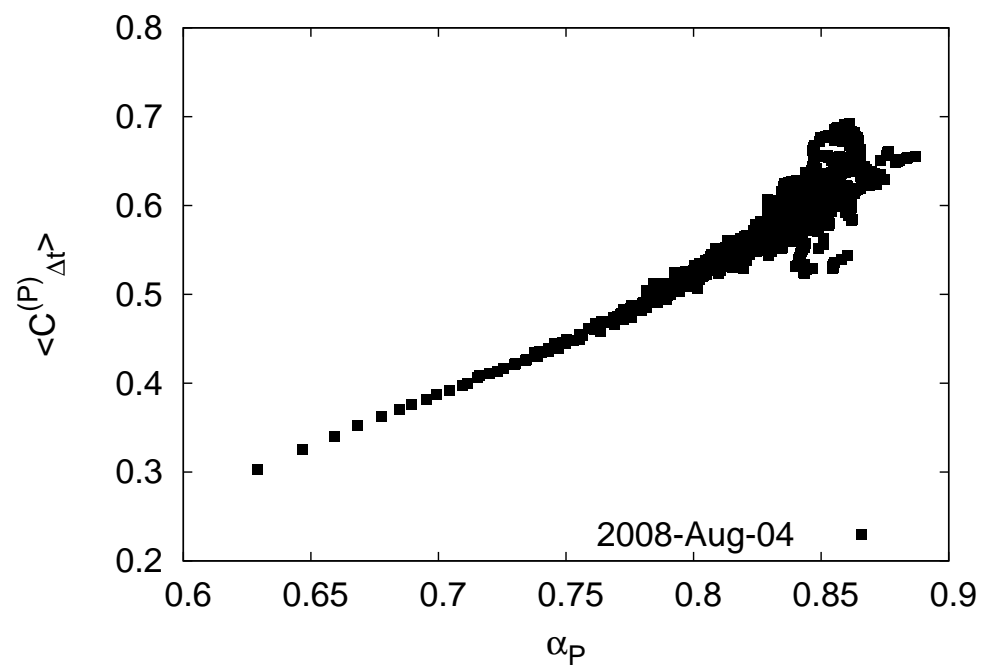

(b)

Fig. 2 (a) The dependence of $\alpha_{P}$ and $\left\langle C_{\Delta t}^{(P)}\right\rangle$ on the values of $\Delta t$. (b) Scatter plots of the scaling exponents $\alpha(\Delta t)$ and the averaged correlation $\left\langle C_{\Delta t}^{(P)}\right\rangle$. The scaling exponents $\alpha_{P}$ and the averaged correlation $\left\langle C_{\Delta t}^{(P)}\right\rangle$ are computed from the quotation activities during a period from 8:38, 3 August 2008, to 21:56, 8 August 2008 (UTC) for the window length $\Delta t \quad(1[\mathrm{~min}] \leq$ $\Delta t \leq 1500[\min ])$.

We compute both the bootstrap mean of the scaling indices and their variance for each week. We generated 1,000 bootstrap samples with 100 points from 10,080 observation points. We computed the temporal variation of scaling exponents $\alpha$ for both quotations and transactions during all the observation weeks. It is found that 


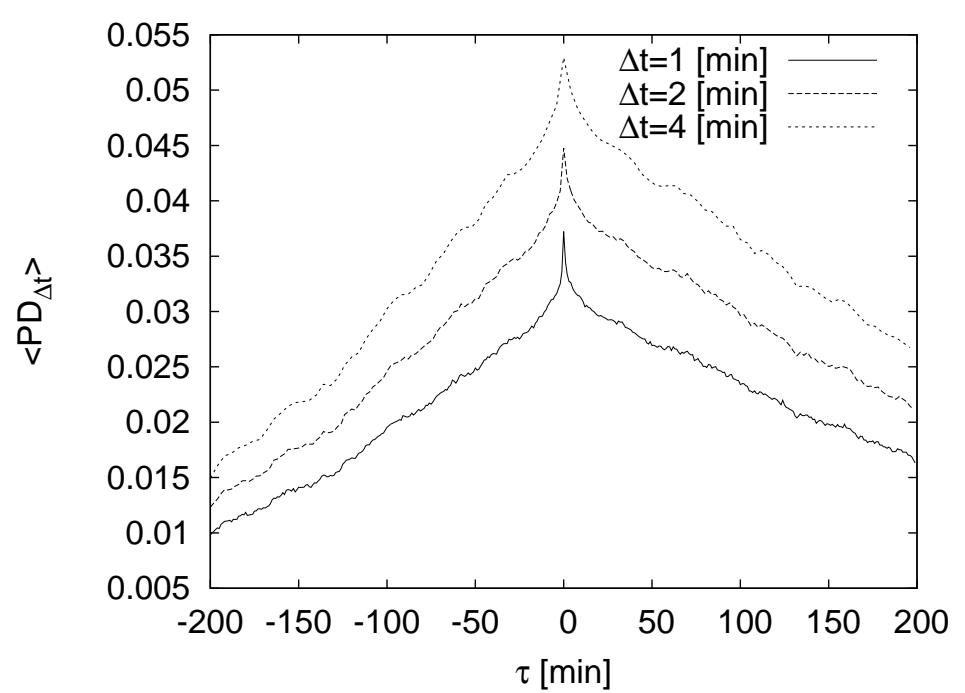

Fig. 3 The global average of cross-correlations between the quotation activities and transaction activities for different time windows. We use the high resolution data during a period from 8:38, 3 August 2008, to 21:56, 8 August 2008 (UTC).

the bootstrap standard deviation of the scaling indices is less than 0.01 . They vary in range from 0.5 to 0.7 .

In order to measure an error between empirical results and a fitting function with the least squars method, we define a norm of residual,

$$
n \operatorname{nrmr}_{X}=\sqrt{\sum_{i=1}^{N}\left(\log \operatorname{Cov}\left(X_{i, \Delta t}, X_{i, \Delta t}\right)(0)-\log \hat{A}_{X}-2 \hat{\alpha}_{X} \log \left\langle X_{i, \Delta t}\right\rangle\right)^{2}}
$$

where $\hat{A}_{X}$ and $\hat{\alpha}_{X}(X=\{P, D\})$ are parameter estimates computed by using the least squared method.

We show the norms of residuals normr $r_{P}$ and normr $_{D}$ for the scaling relationship for each week in Fig. 4. The figures show that the statistical quality of the scaling relationship between the mean values of activities and corresponding standard deviation, the error between the empirical relationships and Eq. (6) with estimates, depends on the observation period.

Let us stress that if the norm of residuals is high then mean activities and their standard deviations may not be fitted to the scaling relation Eq. (6) very well. Specifically, the value of residuals calculated from the number of quotations suddenly increased or decreased before or after the periods of the (I) Paribas shock (Aug. 2007), (II) Bear Stearns shock (Feb. 2008), (III) sub-prime crisis driven by Lehman shock (Sep. 2008 to Mar. 2009) and (IV) Euro crisis (Apr. to May 2010). The scaling relationship seems to break after the (I) Paribas shock but before (III) the sub-prime crisis and (IV) the Euro crisis. The behaviour of residuals computed from the transaction activities is similar. However, the scaling relationship of the transaction activities is more stable than that of the quotation activities before or after the shocks. 
In addition, we compute the global average of the cross-correlation coefficients. The bootstrap standard deviations are approximately 0.1 during all observation periods. We found that the global average of simultaneous cross-correlations for quotation activities takes values less than 0.4 until February 2009. From April to July 2009, it took larger values than before. After July 2009, the value increased and fluctuated around 0.5, while the global average of transaction activities took smaller values. We conclude that the transactions occur in a mutually independent manner.

Figure 5 shows scatter plots of the scaling exponent and the global average of simultaneous cross-correlation matrix. Error bars show a $66.6 \%$ confidence level. The value of the correlation coefficient between $\alpha_{P}$ and $\left\langle C_{\Delta t}^{(P)}\right\rangle$ is 0.464 for quotation activities and that between $\alpha_{D}$ and $\left\langle C_{\Delta t}^{(D)}\right\rangle$ is 0.53 for transaction activities. There is both a relationship between $\alpha_{P}$ and $\left\langle C_{\Delta t}^{(P)}\right\rangle$ and between $\alpha_{D}$ and $\left\langle C_{\Delta t}^{(D)}\right\rangle$. A solid line represents a fitted linear dependence $\left\langle C_{\Delta t}^{(X)}\right\rangle=a_{X} \alpha_{X}+b_{X}$ obtained with the least squared method, where $X=\{P, D\}$. We found that $a_{P}=3.7231, b_{P}=-1.929$ for quotations and $a_{D}=1.0368, b_{D}=-0.4531$ for transactions. The rms of residuals, defined as

$$
r m s_{X}=\sqrt{\sum_{w}\left(\left\langle C_{\Delta t}^{(X)}\right\rangle(w)-a_{X} \alpha_{X}(w)-b_{X}\right)^{2}},
$$

where $\left\langle C_{\Delta t}^{(X)}\right\rangle(w)$ and $\alpha_{X}(w)$ are in the observation week $w$, is 0.0734 for quotations $(X=P)$ and $0.0195(X=D)$ for transactions. It is confirmed that a relationship between the scaling exponent and the global average of cross-correlations is statistically significant.

Moreover, using Eq. (8), we compute the global average of cross-correlations between the quotation activities and transaction activities during the period from June 2007 to December 2010. Since the global average of their simultaneous cross-correlations always takes the maximum value, we compute them at $\tau=0$. Figure 6 shows their global average for each week. The error-bars show a $66.6 \%$ confidence level obtained from the bootstrap standard deviations. The value of $\left\langle P D_{\Delta t}\right\rangle$ is not large but increases eventually. Specifically, after August 2009, the value rapidly increased. This significant increase coincides with scaling breaking of quotation activities. From this result, we conclude that in the EBS brokerage system, the quotation activities changed after August 2009.

\section{Conclusion}

We investigated quotation and transaction activities of the foreign exchange market with 43 months of high resolution data. We examined fluctuation scaling, the global averages of cross-correlation coefficients for both quotation and transaction activities and the global average of cross-correlation coefficients between them.

We found that the scaling relationship between the mean of the numbers of activities and their variance is stable for a long observation period. However, we also observed that the scaling relationship breaks during several periods. Specifically, the scaling breaking was observed from May to June 2009. This may be related to imbalance between demand and supply of the foreign exchange market. The reason for this is an open question. 


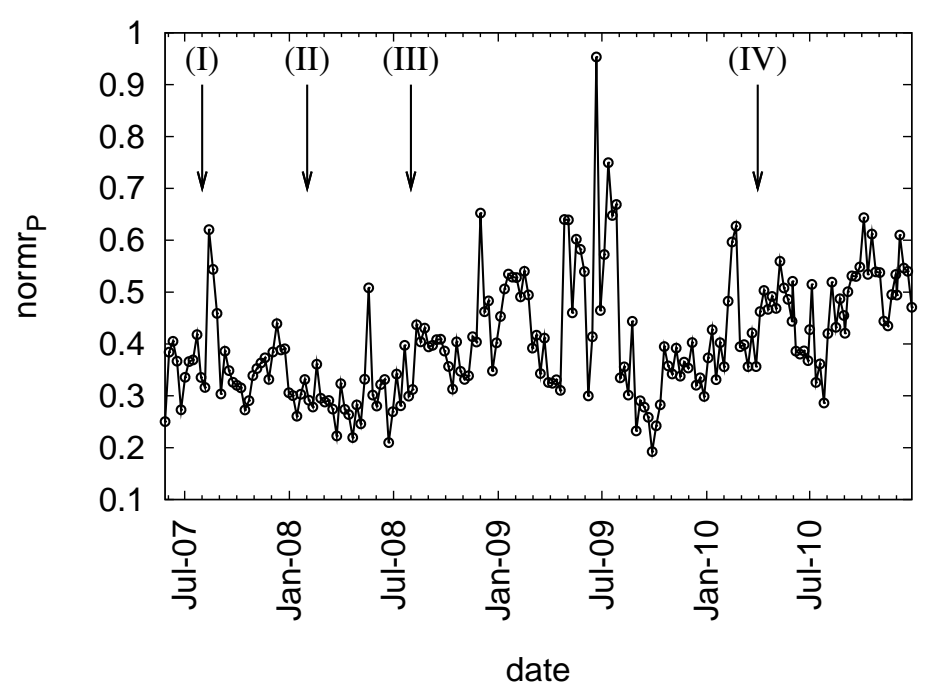

$(\mathrm{P})$

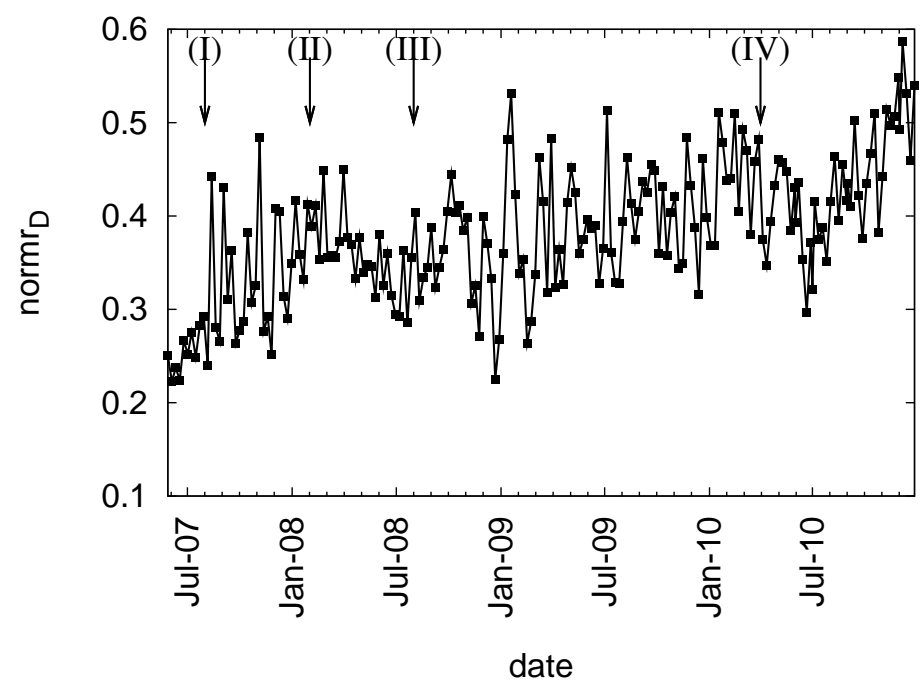

(D)

Fig. 4 The norm of residuals for the scaling relationship between the mean of the number of quotations (P) or transactions (D) and their standard deviation during a period from June 2007 to December 2010.

We further found that there is a relationship between the exponents of fluctuation scaling and the global average of simultaneous cross-correlation coefficients. The relationship was found for both different window lengths and different observation periods. The scaling exponents have a positive association with the global average of cross-correlations. These values may measure the degree of the synchronous behaviour of participants.

Moreover, we recognised that the global average of cross-correlations between the quotation activities and transaction activities rapidly inclined after August 2009. The 


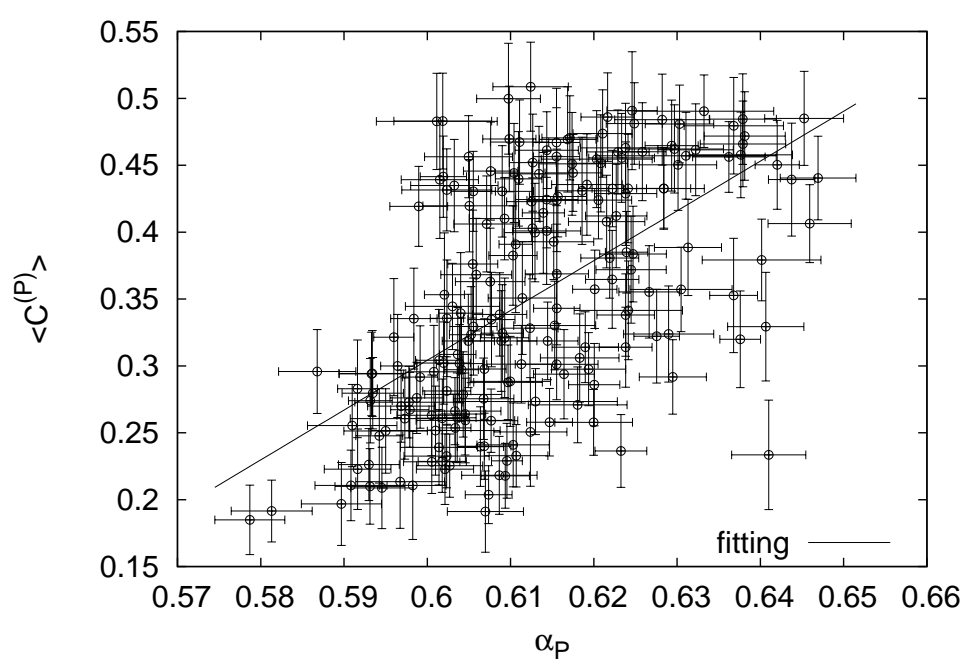

$(\mathrm{P})$

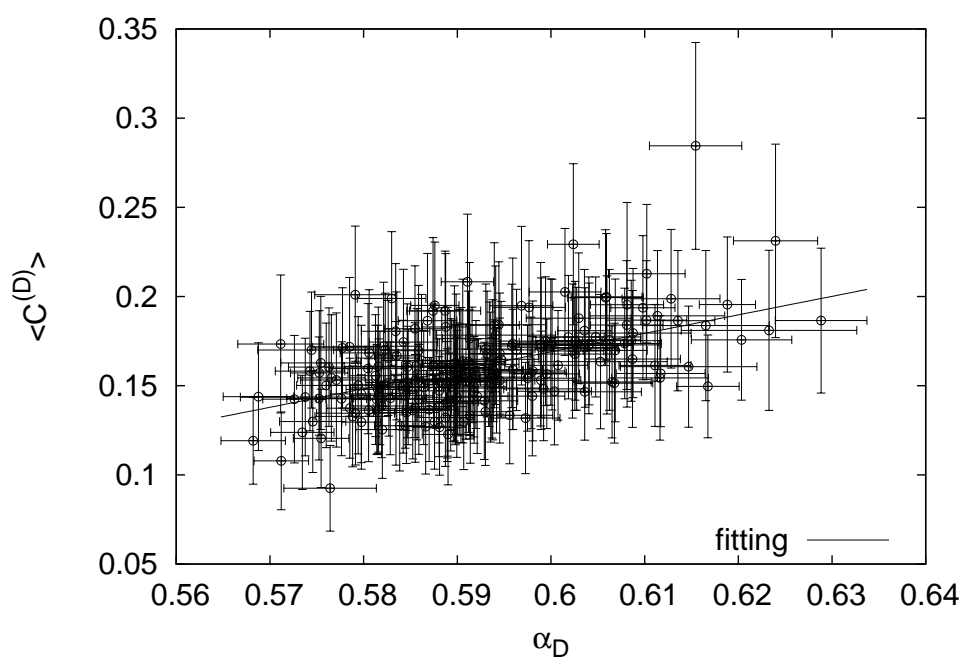

(D)

Fig. 5 Scatter plots between the scaling exponent and global average of simultaneous cross-correlation matrix at the same observation period. (P) is obtained from the number of quotations, and (D) of transactions. The values of the cross-correlation coefficient between $\alpha$ and $\left\langle C_{\Delta t}\right\rangle$ are $0.464(\mathrm{P})$ and $0.53(\mathrm{D})$, respectively. The line represents $\left\langle C_{\Delta t}^{(X)}\right\rangle=$ $a_{X} \alpha_{X}+b_{X} \quad(X=\{P, D\})$.

occurred just after the significant scaling breaking of the quotation activities. The scaling breaking may be related to drastic changes in participants' perception of the market.

In future research, the relationship between trends of exchange rates and scaling exponents could be explored. The link between the intuitive understanding of the 


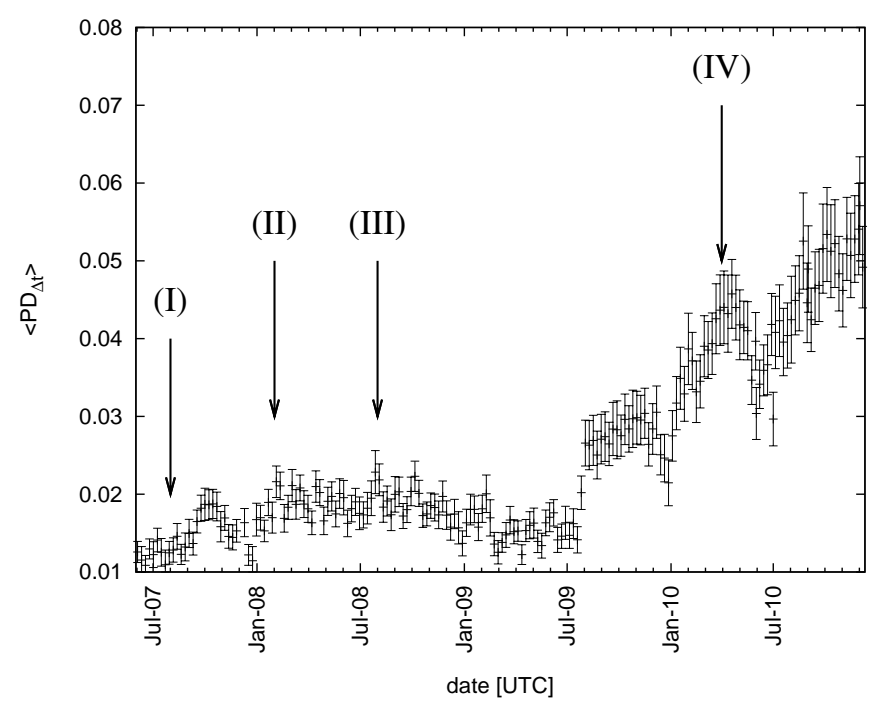

Fig. 6 The global average of cross-correlations between the quotation activities and transaction activities during the period from June 2007 to December 2010.

prevailing market state by market participants and estimated scaling exponents should be examined.

Acknowledgements This work was supported by the Grant-in-Aid for Young Scientists (B) by Japan Society for the Promotion of Science (JSPS) KAKENHI (\#23760074) (A.-H. Sato). It was also financially supported by the Japan Securities Scholarship Foundation, Grants-in-Aid for Scientific Research (A), Ministry of Education, Culture Sports, Science and Technology (No. 21243019), and by Ishii Memorial Securities Research Promotion Foundation (T. Hayashi). This was also supported by the European COST Action MP0801 Physics of Competition and Conflicts and by the Polish Ministry of Science Grant 578/N-COST/2009/0 (J.A. Holyst).

\section{References}

1. New IT Infrastructure for the Information-explosion Era (July 2005-March 2011), http://www.infoplosion.nii.ac.jp/info-plosion/ctr.php/m/IndexEng/a/Index/

2. Drożdż S, Kwapień J, Oświẹcimka P, Rak R (2010) The foreign exchange market: return distributions, multifractality, anomalous multifractality and the Epps effect. New J Phys 12:105003

3. Rebitzky RR (2010) The influence of fundamentals on exchange rates: Findings from analyses of news effects. J Econ Surv 24:680

4. Kaltwasser PR (2010) Uncertainty about fundamentals and herding behavior in the FOREX market. Physica A 389:1215

5. Alfarano S, Lux T, Wagner F (2006) Estimation of a simple agent-based model of financial markets: An application to Australian stock and foreign exchange data. Physica A 370:38

6. Gworek S, Kwapień J, Drożdż S. (2010) Sign and Amplitude Representation of the Forex Networks. Acta Phys Pol A 117:681

7. Liu LZ, Qian XY, Lu HY (2010) Cross-sample entropy of foreign exchange time series. Physica A 389:4785

8. Sazuka N, Inoue J, Scalas E (2009) The distribution of first-passage times and durations in FOREX and future markets. Physica A 388:2839 
9. Inoue JI, Sazuka N Queueing (2010) Theoretical analysis of foreign currency exchange rates. Quant Financ 10:121

10. Ohnishi T, Mizuno T, Aihara K, Takayasu M, Takayasu H (2004) Statistical properties of the moving average price in dollaryen exchange rates. Physica A 344:207

11. Hashimoto Y, Ito T (2010) Effects of Japanese macroeconomic statistic announcements on the dollar/yen exchange rate: High-resolution picture. J Jpn Int Econ 24:334

12. Kyle AS (1985) Continuous Auctions and Insider Trading. Econometrica 53:1315

13. Bonanno G, Lillo F, Mantegna RN (2000) Dynamics of the number of trades of financial securities. Physica A 280: 136

14. Mandelbrot B, Taylor HM (1967) On the distribution of stock price differences. Oper Res 15:1057

15. Clark PK (1973) A subordinated stochastic process model with finite variances for speculative prices. Econometrica 41:135

16. Tauchen T, Pitts M (1983) The price variability-volume relationship on speculative markets. Econometrica 51:485

17. Richardson M, Smith T (1994) A direct test of the mixture of distributions hypothesis: Measuring the daily flow of information. J Financ Quant Anal 29:101

18. Karpoff JM (1987) The relation between price changes and trading volume: A survey. J Financ Quant Anal 22:109

19. Ane T, Geman H (2000) Order Flow, Transaction Clock, and Normality of Asset Returns. J Financ 55:2259.

20. Fergusson K, Platen E (2006) On the distributional characterization of daily log-returns of a world stock index. Appl Math Financ 13:19

21. Beck C (2004) Superstatistics: Theory and applications. Continuum Mech Therm 16:293

22. Miccichè S, Bonanno G, Lillo F, Mantegna RN (2002) Volatility in financial markets: stochastic models and empirical results. Physica A 314:756

23. Podobnik B, Horvatic D, Petersen AM, Njavro M, Stanley HE (2010) Common scaling behavior in finance and macroeconomics. Eur Phys J B 76:487

24. Wang F, Shieh SJ, Havlin S, Stanley HE (2009) Statistical analysis of the overnight and daytime return. Phys Rev E 79:056109

25. Eisler Z, Kertész J (2006) Scaling theory of temporal correlations and size-dependent fluctuations in the traded value of stocks. Phys Rev E 73:046109.

26. Taylor LR (1961) Aggregation, variance and the mean. Nature189:732

27. de Menezes MA, Barabási A-L (2004) Fluctuations in network dynamics. Physical Review Letters 92:028701

28. Eisler Z, Bartos I, Kertész J (2008) Fluctuation scaling in complex systems: Taylor's law and beyond. Adv Phys 57:89

29. Mantegna RN (1999) Hierarchical structure in financial markets. Eur Phys J B 11:193

30. Podobnik B, Horvatic D, Petersen AM, Stanley HE (2009) Cross-correlations between volume change and price change. P Natl Acad Sci USA 106:22079

31. Horvatic D, Stanley HE, Podobnik B (2011) Detrended cross-correlation analysis for nonstationary time series with periodic trends. Europhys Lett 94:18007

32. Duan WQ, Stanley HE (2011) Cross-correlation and the predictability of financial return series. Physica A 390:290

33. Bonanno G, Caldarelli G, Lillo F, Miccichè S, Vandewalle N, Mantegna RN (2004) Networks of equities in financial markets. Eur Phys J B 38:363

34. The data is purchased from ICAP EBS: http://www.icap.com

35. Efron B (1979) Bootstrap methods: Another look at the Jackknife. Ann Stat 7:1

36. Sato A-H, Nishimura M, Hołyst JA (2010) Fluctuation scaling of quotation activities in the foreign exchange market. Physica A 389:2793 